\title{
Research on Engagement Curve of Clutch Based on Optimization Theory
}

\author{
Bing Long ${ }^{1, \text { a }}$ \\ ${ }^{1}$ Nanchang Institute of Science \& Technology, Nanchang, 330108, China \\ a455374361@qq.com
}

Keywords: engagement curve; optimization theory; optimal control

\begin{abstract}
Clutch is a commonly used component in mechanical transmission. It is in the flywheel housing of engine and gearbox, which can separate or engage the transmission system at any time, and transmit the output power of engine. This paper optimizes the clutch engagement curve based on the optimization theory. The results show that the new curve obtained by the correction is significantly lower than the original curve from the aspects of the sliding grinding work and the impact degree.
\end{abstract}

\section{Introduction}

The basic function of the automobile transmission system is to transmit the engine's power to the driving wheel [1]. The clutch is a component that is directly connected with the engine in the automobile transmission system. Before starting a car, it is necessary to start the engine. Then, the transmission should be in neutral position, and the connection between the engine and the driving wheel will be disconnected to remove the engine load. After the engine has been started and started to operate at a normal speed, the transmission can be put on a certain gear to make the car start. When the car starts, the car is gradually accelerating from a state of complete rest. If the engine from the drive train rigid links, the transmission of a hanging file, the car will forward suddenly impulse, but failed to start. This is because the car produces a lot of inertia force when it comes from still to the front. The engine causes great resistance to the engine. Under the action of inertia resistance moment, the engine suddenly drops to the lowest speed below the instantaneous speed, so the engine can't work and can't start. The primary function of the clutch is to ensure a smooth start of the car. In the transmission system is provided with a clutch, after the start of the engine, the car started before the driver to the clutch pedal, the clutch engagement gradually, gradually in the clutch engagement process, the engine torque is also blocked increases gradually, it should also speed up the pedal down gradually, gradually increase the engine's fuel supply. Always keep the engine speed above the minimum stable speed, will not die. Due to the tightness of the clutch engagement, the torque of the engine through the drive train to the driving wheel is increasing. When the traction force is enough to overcome the starting resistance, the car begins to move from rest and gradually accelerates. Another function of the clutch is to ensure the smooth work of the transmission gear shift. In the process of automobile exercise, to adapt to the changing conditions of exercise, the transmission system often needs to be replaced by different gears. To achieve gear shift, gear shift is usually done by shifting gears or other gear mechanisms, so that a gear pair of the original gear stops from the transmission, and the gear pair of the other gear enters the work [2].

\section{Summary of Optimization Theory}

\subsection{Optimization Question}

Optimization is an important branch of Applied Mathematics, optimization can be defined as a mathematical method, it can be used for planning of various production activities in the available resources (resources refers to the mineral resources, hydropower, manpower, equipment, raw materials, transportation conditions, ecological environment, capital, time and space constraints etc.) next, make the production activities or obtain the maximum benefit with the least resources to 
complete the assigned production activities. According to the constraint conditions and whether the objective function is a linear function classification, it is divided into linear programming and nonlinear programming. When the objective function is linear function, and the constraint condition is determined by linear equality function and linear inequality function, this kind of problem is called linear programming. The study of nonlinear programming is the problem of having nonlinear functions in the objective function or constraint function. When the objective function is a two function and the constraints are determined by linear equality function and linear inequality function, it is called the two-degree programming. The optimization method is formed in recent decades. It mainly applies mathematical methods to study the optimization ways and schemes of various optimization problems, and provides scientific decision basis for policymakers. The main research object of the optimization method is the management problems of all kinds of organized systems and their production and operation activities. The purpose of the optimization method is to find a best plan for rational use of manpower, material and financial resources for the studied system, and to bring into play and improve the efficiency and effectiveness of the system, and ultimately achieve the best goal of the system. Practice shows that, with the development of science and technology progress and production operation, the optimization method has become an important theoretical basis and indispensable part of the modern management science, it is widely applied to public management, economic management, engineering construction, national defense and other fields, playing an increasingly important role [3].

\subsection{Solutions to Optimization Question}

The basic method to solve the linear programming problem is simplex method, is the most thorough one direction, and has one of the most widely used is still the best algorithm, standard software has simplex method, in the electronic computer to solve the constrained conditions and the number of decision variables of the linear programming problem. Its theoretical basis is that the feasible domain of linear programming is the multifaceted convex set in n-dimensional vector space $\mathrm{Rn}$, and its optimal value must be reached at the vertex of the convex set if it exists. The feasible solution corresponding to the vertex is called the basic feasible solution. The basic idea of simplex method is to find out a basic feasible solution first and identify it to see if it is the best solution. If not, then transform it to another basic feasible solution according to certain rule, then identify it; if it is not, then transform it and repeat it according to this rule. As the number of basic feasible solutions is limited, the optimal solution of the problem can be obtained by the finite transformation. If the problem has no optimal solution, it can be judged by this method. The general solution steps of simplex method can be summarized as follows: the constrained equations of linear programming problem are expressed as canonical equations, and the basic feasible solution is found as the initial base feasible solution. If the basic feasible solution does not exist, that is, the constraint conditions are contradictory, then the problem has no solution. If the basic feasible solution exists, starting from the initial basic feasible solution, according to the optimality condition and the feasible condition, we introduce a non-base variable instead of a base variable to find another feasible solution of the objective function value. Iterates until the corresponding test number satisfies the optimality condition, that is, the optimal solution of the problem is obtained. If the target function value of the problem is unbounded during the iterative process, the iteration is terminated.

The solution of nonlinear programming problem is generally much more difficult than linear programming, and there is no general algorithm suitable for all kinds of nonlinear problems at present. Each algorithm has its own specific application scope. In some cases, for the convenience of calculation, the nonlinear programming problem will be approximated to a linear programming problem. The significance of unconstrained method is that although most practical programming problems are constrained, many constrained optimization methods can transform constrained problems into some unconstrained problems. Most of the unconstrained optimization methods are iterative algorithms for one - step one - dimensional search. This kind of iterative algorithm can be divided into two types. A class of guide functions which need to be used by the objective function is called the analytic method. The other kind does not involve the derivative, which is used only to the 
function value, which is called the direct method. The basic idea of these iterative algorithms is to select a favorable search direction at one approximate point, and search for a new approximate point in this direction. The same procedure is then performed on the new point to iterate again and again until the desired precision is met. According to the different search direction, there can be a variety of algorithms. The analytic algorithms are: the gradient method, also known as the steepest descent method. This is an early analytical method, and the convergence rate is slow. Newton method: the speed of convergence is fast, but it is not stable, and it is also difficult to calculate. Conjugate gradient method: the convergence is faster and the effect is better. Variable scale method: This is a kind of efficient method. The method is the most commonly used method. The algorithms of direct type include alternating direction method, pattern search method, rotation direction method, Powell conjugate direction method and simplex acceleration method. Line direction: This is a class of iterative algorithms that can approximate the most advantages by selecting the feasible descent direction by one by one. Such as Tandy g, Frank Wolf, projection gradient and reduced gradient are all such algorithms. Approximate algorithm: these algorithms include sequential linear programming and sequential two - order programming. The former turns the original problem into a series of linear programming problems, and the latter turns the original problem into a series of programming problems [4].

\section{Optimization of Engagement Curve of Clutch}

\subsection{Slipping State Modeling}

In the modeling of the separation state, the basic module appears, and the module is used for the jump of the signal, indicating the source and direction of the signal. We should define the tag visibility as a global variable. Otherwise, the operation result is wrong. The model is built with Enable System, even if the subsystem can be built. The characteristic of the model is that when the control signal of the enable port is positive, the subsystem can be executed; otherwise, the subsystem will not execute. The driven disk has great influence on the clutch's working performance. The design should meet the following requirements: the inertia moment of the driven plate should be as small as possible to reduce the impact between the gears of the gearbox when shifting. The driven disc should have axial elasticity, so that the clutch is smooth and easy to start, and the pressure of the friction surface is uniform to reduce the wear. The torsional vibration damper should be installed to avoid the resonance of the transmission system and to ease the impact. It should have enough stiffness, otherwise it will affect the working characteristics of the clutch, increase the separation stroke during operation and reduce the lift of the pressure plate, so that the friction surface cannot be completely separated. It should be in good alignment with the flywheel so as not to affect the balance of the assembly and the normal work. The diaphragm spring support should have high dimensional precision. To facilitate the ventilation and heat dissipation and prevent the high temperature of the friction surface, a larger ventilation window hole can be opened on the clutch cover, or the fan sheet is added to the cover. The separation bearing assembly is composed of the separation bearing and the separation sleeve. The separation bearing is mainly subjected to axial separation force when working, and it also bears the radial force under the action of centrifugal force at the time of high speed rotation. At present, the domestic automobile uses more angular contact thrust ball bearings, with full sealed structure and high temperature lithium base grease, the face shape and is matched with the shape of the tip of fingertip, with spherical end surface is a plane, the tip curved surfaces with flat end face or concave curved face.

\subsection{Locked State Modeling}

When it is in the joint state, the friction torque required to maintain the clutch engagement is greater than the maximum static friction torque. The maximum static friction torque is not enough to transmit such a large torque, then switch to the sliding state. The pressure plate should have larger mass to increase the heat capacity and reduce the temperature, prevent cracks and breakages, and sometimes set various shapes of heat dissipation bars or blast ribs to help heat dissipation and 
ventilation. The middle pressure plate can be cast out the ventilator, and the aluminum alloy pressure plate with large heat transfer coefficient can be used. The pressure plate should have larger rigidity, make the pressure distribution of the pressing force on the friction surface even and reduce the warping deformation after heating, so as not to affect the even compression of the friction disc and the complete separation from the clutch. And the flywheel should be kept in good alignment, and the static balance should be carried out, and the balance accuracy of the single piece of the press plate should be. The height tolerance of the press plate is small. A more complex shape, requirements of heat transfer is good, the friction coefficient is high, usually using grey cast iron, clutch, flywheel drive clutch cover platen drive rotates, and the friction between the platen and the driven disc friction plate of the driven wheel; in the clutch separation, pressure free axial movement relative to the clutch cover so, the driven disc release. These movements are all done by the transmission. The two ends of the drive plate relate to the clutch or bolt respectively with the clutch cover and the press plate, and the circumferential arrangement is generally adopted. When the clutch is engaged, the clutch cover drives the platen to rotate together through it. When the clutch is separated, it can make use of its elastic restoring force to affect the axial separation of the pressure plate and reduce the maneuvering force. Clutch cover and engine flywheel bolt together when the diaphragm spring is pre-pressed, clutch is in an engaged position, the pressing force of diaphragm spring pressure plate, causing friction between the plate and the driven friction plate. When the clutch cover assembly rotates with the flywheel, the driven disc assembly rotates together with the gearbox to transfer engine power through the friction torque on the friction disc.

\subsection{Comparison of Engagement Curves of Clutch before and after Optimization}

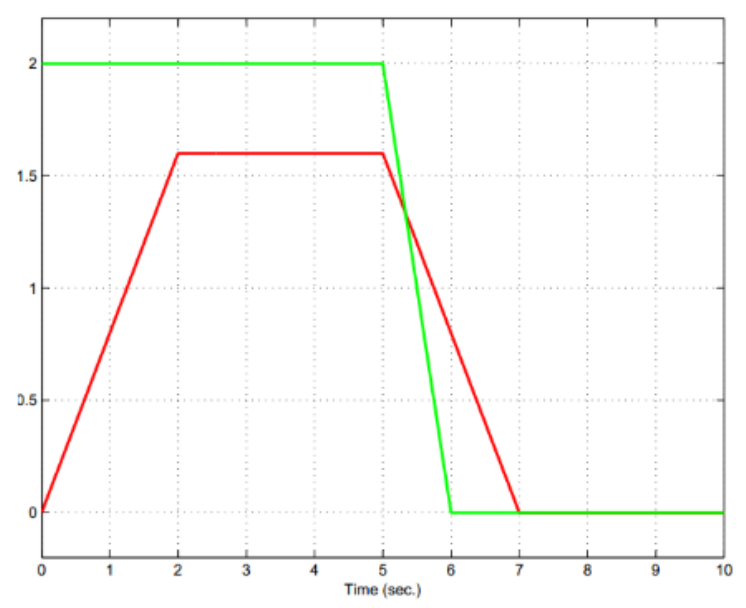

Figure 1. Engagement curve of clutch before optimization

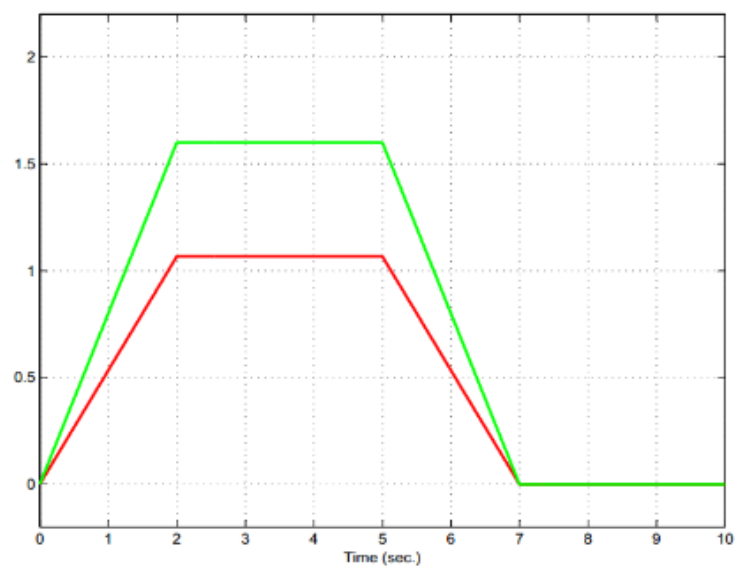

Figure 2. Engagement curve of clutch after optimization 


\section{Conclusion}

From the curve of joint trend can be seen in line with the characteristics of the clutch engagement, which is proved that this algorithm can reduce the friction work and good effect of reducing the degree of impact. In practical application, the original curve can be determined according to the actual operation of the driver, and by optimizing joint correction the curve of AMT clutch, so that does not change the basic requirements of joint under the condition of AMT to reduce the impact and the friction work.

\section{References}

[1] Lu You, Zhu Xiaoliang, Li Xiaoqing, et al. Evaluation Feeling Hiding in Clutch Performance Curve [J]. Automobile Parts, 2015(6): 35-37.

[2] Zhang Kunxiao, He Haiming, Wu Dongsheng. Study on Optimization Design of Clutch Spring Based on Objective Function in MATLAB [J]. Automobile Parts, 2012(5): 73-75.

[3] Jin Peng, Zhang Guogeng, Song Wei. The Optimization of Spring Characteristic Curve of Clutch Pedal [J]. Internal Combustion Engine \& Powerplant, 2014, 31(5): 4-6.

[4] Peng Jianxin, Jin Hui, Chen Huiyan. Engagement Curve of Clutch Based on Optimization Theory [J]. Transactions of Beijing Institute of Technology, 2009, 29(11): 964-967+971. 